\title{
Context preexposure prevents forgetting of a contextual fear memory: Implication for regional changes in brain activation patterns associated with recent and remote memory tests
}

\author{
Joseph C. Biedenkapp and Jerry W. Rudy ${ }^{1}$ \\ Department of Psychology, Center for Neuroscience, University of Colorado, Boulder, Colorado 80309, USA
}

\begin{abstract}
Contextual fear conditioning was maintained over a 15-day retention interval suggesting no forgetting of the conditioning experience. However, a more subtle generalization test revealed that, as the retention interval increased, rats showed enhanced generalized fear to an altered context. Preexposure to the training context prior to conditioning, however, prevented this enhanced generalized fear from developing. These results support the hypothesis that the memory representation of the context degrades as the memory ages and is responsible for enhanced generalization. The implications of these results for systems consolidation versus forgetting interpretations of regional changes in neural activation patterns that occur as memories age are discussed.
\end{abstract}

What happens to a memory as it ages? One influential idea is that memories that initially depend on the hippocampus undergo what is called systems consolidation (Squire et al. 1984; Teyler and DiScenna 1986; McClelland et al. 1995; Squire and Alvarez 1995). The hippocampus, by its interactions with neocortical sites, is thought to participate in the strengthening of connections among these cortical sites so that they can now support the retrieval of the memory without any contribution from the hippocampus. In order for such a systems consolidation process to work, however, it must operate in the face of another countervailing force-forgetting. As a memory ages, either due to decay or interference introduced by additional experience, information in the trace is likely to degrade.

The relationship between forgetting and performance, however, is complicated. The most direct relationship would be that as forgetting occurs retention performance would be impaired. A number of years ago, however, Riccio and colleagues (Riccio and Ebner 1981; Riccio et al. 1992) called attention to another way in which forgetting can manifest itself. Shortly after a conditioning experience animals are very good at discriminating between the actual training context and an altered context that contains only a subset of the features of the training context. Thus, the training context evokes more conditioned responding than the altered context. In this case the generalization gradient is said to be quite sharp. However, the generalization gradient broadens when the retention interval is lengthened. So, now the altered context evokes significantly more responding. Note that in this case there is no loss in the ability of the training context to evoke conditioned responding. Instead, more responding is evoked by the test context.

Riccio et al. (1992) have interpreted this enhanced generalization to reflect some degradation of the original memory trace so that animals become uncertain about the details of the original experience. This idea may be relevant to interpreting some of the results that can be considered to support the idea that as memories age they become independent of the hippocampus.

\footnotetext{
'Corresponding author.
}

E-mail JRudy@Clipr.Colorado.Edu; fax (303) 492-2967.

Article is online at http://www.learnmem.org/cgi/doi/10.1101/lm.499407.
For example, Bontempi, Frankland, and colleagues (Bontempi et al. 1999; Frankland et al. 2004; Maviel et al. 2004) measured neuronal activity in different brain regions induced by a retention test of memories thought to initially depend on the hippocampus. Their important finding was that activity in the hippocampus and regions of the prefrontal cortex (PFC) changed significantly as a function of the age of the memory. For recently acquired memories, there was high activity in the hippocampus but low activity in the PFC regions. The opposite pattern was observed for older memories. Frankland and Bontempi (2005) interpreted these results in the context of the standard theory of systems consolidation, suggesting that PFC regions play the same role in retrieving "old" memories as the hippocampus plays in retrieving "new" memories. Thus, memories initially supported by the hippocampus become consolidated into the PFC and other cortical areas, with the PFC being a critical locus orchestrating retrieval after consolidation because of its broad patterns of interconnectivity with other brain areas.

In a commentary, we suggested an alternative accountthat these regional changes in neural activity occurring as the memory ages reflect the process of forgetting (Rudy et al. 2005). We suggested that old memories (1) are degraded and more difficult to retrieve than recent memories, and (2) require additional activation of the PFC to be retrieved. On this view, the enhanced activity in PFC reflects the impact of forgetting, not systems consolidation. Our account is consistent with several contemporary views of the contribution PFC makes to retrieval (O'Reilly et al. 1999; Botvinick et al. 2001; Fletcher and Henson 2001; Miyashita 2004; Ranganath et al. 2004).

Frankland and Bontempi arrived at their theoretical conclusions from studies of contextual fear conditioning (Frankland et al. 2004) and a food-reinforced based place learning task (Maviel et al. 2004). Consistent with our interpretation, Maviel and colleagues reported some evidence of forgetting of the correct place response on the remote memory test. However, Frankland et al. (2004) reported no evidence of forgetting in their contextual fear experiments.

Without evidence of forgetting in the contextual fear task, it is clearly speculative for Rudy et al. (2005) to claim that enhanced neural activity in PFC was due to the demand of retrieving a degraded old memory. Frankland et al. (2004), however, 
only assessed retention by comparing conditioning evoked by the training context as a function of the age of the memory. The purpose of our experiments was to determine if the more subtle, enhanced-generalization, measure of forgetting would reveal that the contextual fear memory degrades as it ages.

The goal of the first experiment was to determine if generalized contextual fear conditioning increases as the memory trace ages. As shown in Figure 1, when the retention interval was only $1 \mathrm{~d}$ rats displayed more freezing in training context, $\mathrm{A}$, than in the generalization context, $\mathrm{B}, F_{(1,9)}=5.8, P<0.05$. However, when the retention interval was 7 or $15 \mathrm{~d}$ the difference in the amount of freezing in the two contexts was not significantly different $(P>15$ and 0.36 respectively). Thus, generalized fear increased as the retention interval increases.

If the results of the first experiment are due to the memory trace degrading, then it should be possible to prevent the increase in generalized fear by preventing the memory trace from degrading. Contextual-fear conditioning is thought to be a product of two learning processes: (1) as a consequence of exploring the context, the rats acquire what is called a conjunctive or configural representation of the context, and (2) this representation is associated with the shock (Fanselow and Rudy 1998; Rudy et al. 2004). According to this view the hippocampus binds the cooccurring features of the context into a unique representation that can then associate with shock. Enhanced generalization that results as the retention interval increases would occur because the conjunctive trace degrades so the rat can no longer discriminate Context $\mathrm{B}$, used to test for generalization, from Context $\mathrm{A}$, the training context.

If this account is correct then if a stronger representation of the training context is established, it should be possible to prevent enhanced generalized fear that occurs as the memory trace ages. In order to produce a more stable representation of the training context, on three successive days prior to context-shock training we preexposed rats to Context A for $5 \mathrm{~min}$. To equate for nonspecific effects of context preexposure, control subjects were exposed to a much different context, Context X. After conditioning to Context $\mathrm{A}$, rats were then tested either 1 or $15 \mathrm{~d}$ to either Context A or to Context B, the altered context. We predicted that preexposure to the training context would prevent rats from displaying enhanced generalized fear to Context B. The three contexts used in this experiment are illustrated in Figure 2A.
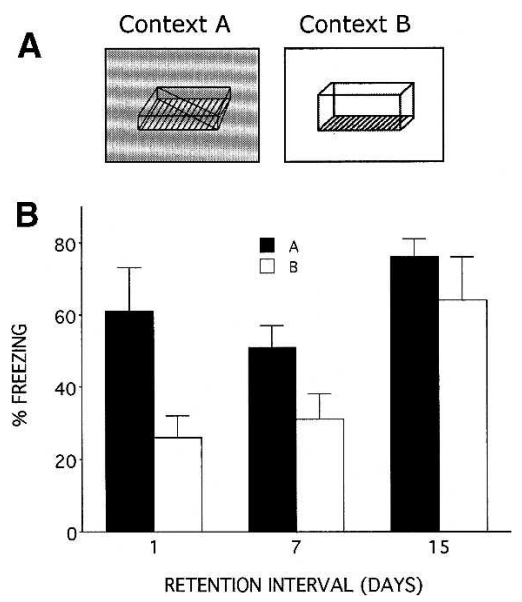

Figure 1. (A) Illustration of the two contexts used in the first experiment. Context A was the training context and Context B was the generalization test context. $(B)$ Mean percentage freezing as a function of retention interval. Rats displayed significantly more freezing to Context $\mathrm{A}$ than to Context B, but only when the retention interval was $1 \mathrm{~d}$. Error bars represent SEM.
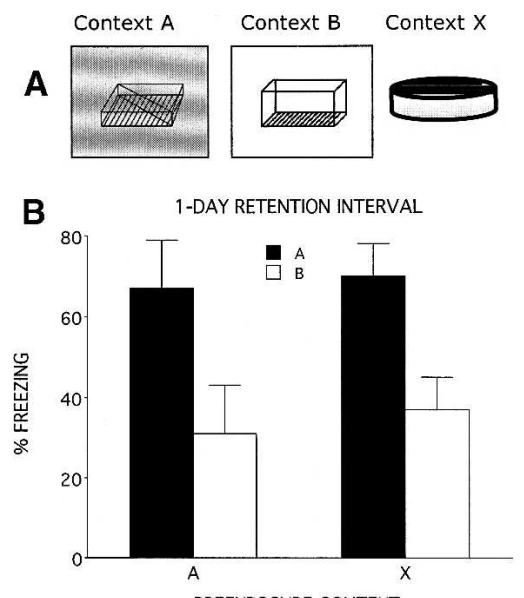

PREEXPOSURE CONTEXT

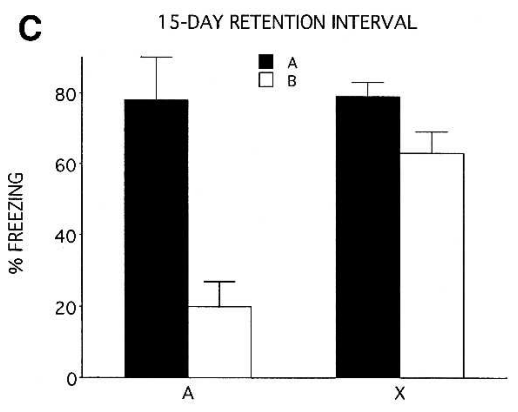

PREEXPOSURE CONTEXT

Figure 2. (A) Illustration of the three contexts used in the second experiment. Context A was the training context and Context B was the generalization test context. Rats were either preexposed to Context A or Context X. (B) The mean percentage freezing when the retention interval was $1 \mathrm{~d}$. Regardless of preexposure condition, rats displayed significantly more freezing to Context A than to Context $\mathrm{B}$. (C) The mean percentage freezing when the retention interval was $15 \mathrm{~d}$. Rats preexposed to Context A displayed significantly more freezing to Context A than to Context $B$, but rats preexposed to the control context, $X$, displayed the same level of freezing in both contexts. Error bars represent SEM.

Figure $2 \mathrm{~B}$ presents the results of the 1-d retention test. Irrespective of whether rats were preexposed to the training context, $\mathrm{A}$, or to the control context, $\mathrm{X}$, rats displayed more freezing to Context A than to Context B. A two-factor analysis of variance (Preexposure Context and Test Context) revealed only a main effect of Test Context, $F_{(1,20)}=11.87, P<0.05$. The main effect of Preexposure Context and the Preexposure versus Test Context interaction were not significant, $F<1$.

Test performance at the 15-d interval (Fig. 2C) was strongly influenced by context preexposure. Rats preexposed to the control context, $\mathrm{X}$, showed more freezing to the generalization test context, $\mathrm{B}$, than did rats preexposed to the training context, $\mathrm{A}$. In addition, rats preexposed to training context, $\mathrm{A}$, displayed much less freezing in Context B than they did in Context A. However, rats preexposed to Context $\mathrm{X}$ displayed as much freezing in the Context $\mathrm{B}$ as they did in Context A. Consistent with this description, a two-factor ANOVA on these data reveals a main effect of Preexposure Context, $F_{(1,20)}=7.4, P<0.01$, Text Context, $F_{(1.20)}=21.9, P<0.0001$, and a significant Preexposure $X$ Test Context interaction, $F_{(1,20)}=6.8, P<0.02$. Post-hoc analysis of the interaction revealed that rats preexposed to the training context froze significantly more in Context A than in Context B $(P<0.01)$, but rats exposed to the control context did not $(P>0.15)$. Moreover rats preexposed to the control context froze more in Context $\mathrm{B}$ than rats preexposed to the training context. 
The results of the second experiment are particularly important in distinguishing between the consolidation and forgetting accounts of the effects of retention on memory. This is because they reveal that preexposure to the training context, which should result in a stronger memory representation of the context, prevented enhanced generalized fear that normally occurs with longer retention intervals. The results support the hypothesis that enhanced generalization seen at the 15 -d retention interval was due to forgetting.

The results of the second experiment also rule out another interpretation of the enhanced generalization effect. One might argue that enhanced generalized fear is the product of some fear incubation process that occurs over time (Eysenck 1968). This general increase in fear would then disproportionately increase the level of fear to the generalized test context, thus producing enhanced generalization. The results of the second experiment rule out this hypothesis because the fear incubation account cannot explain why preexposure to the training context prevents enhanced generalized fear from occurring.

The present results are consistent with those reviewed by Riccio et al. (1992), indicating that generalization gradients broaden as the retention interval increases. They are also consistent with Riccio et al.'s (1992) general interpretation that enhanced generalization is the result of a degrading memory trace (i.e., forgetting). They strengthen this view, however, because they reveal that a learning experience (preexposure to the training context), which can be reasonably assumed to strengthen the context representation (and thus its resistance to degradation), prevented enhanced generalization. We also note that brief reminder exposures to a training context after training can resharpen the generalization gradient (Zhou and Riccio 1994; Rosas and Bouton 1997). Such data are also consistent with the hypothesis that the broadening of the generalization gradient represents forgetting.

Our experiments support the view that as context memories age the conjunctive trace supported by the hippocampus degrades, and this leads to a broader generalization gradient. The hippocampus makes a major contribution to contextual fear conditioning in intact animals. However, it should be appreciated that other regions of the brain can support contextual fear conditioning when the hippocampus is removed prior to conditioning (Wiltgen et al. 2006; see Maren 2001; Rudy et al. 2005). One implication of our hypothesis is that rats with anterograde damage to the hippocampus and thus lack of conjunctive representation of context should display more generalized contextual fear conditioning than intact rats. There are several reports that are consistent with this implication (Frankland et al. 1998; Antoniadis and McDonald 2000). For example Antoniadis and McDonald reported that rats with anterograde damage to the hippocampus display contextual fear conditioning but have difficulty discriminating between the shocked context and another similar context. Consistent with our view, Antoniadis and McDonald (2000) proposed that the hippocampus is important for discriminating between contexts that share a number of features.

As we noted (Rudy et al. 2005), if a memory trace degrades as the retention interval increases, it is difficult to interpret regional changes in brain activation that occur as the memory trace ages (see Frankland and Bontempi 2005). One possibility is that such changes reflect some form of systems consolidation (Frankland and Bontempi 2005) in which the memory trace becomes independent of the hippocampus and consolidated in the PFC. The experiments just described, which reveal substantial forgetting of the contextual memory trace, are consistent with our general contention (Rudy et al. 2005) that such regional changes reflect the brain's attempt to deal with forgetting. Initial reports examining the effects of hippocampus lesions on the re- trieval of contextual fear memories supported the systems consolidation hypothesis because they found remote but not recent memories were spared by damage to the hippocampus (Kim and Fanselow 1992; Anagnostaras et al. 1999). However, damage to the hippocampus was limited to only the dorsal region. Recently, it has been reported that complete lesions of the hippocampus spare neither recent nor remote fear memories (Lehman et al., in press). This result thus challenges the systems consolidation interpretation of the effect of memory age on regional changes in brain activation patterns. Our forgetting interpretation, however, is consistent with these recent data.

Long-Evans rats 70-80 d old were used in these experiments. They were housed four to a cage at $25^{\circ} \mathrm{C}$ on a 12 -h light/dark cycle. Rats received ad lib food and water. There were five or six rats in each of the groups in the first experiment and six rats in each group in the second experiment. All procedures were conducted in accordance with protocols approved by the University of Colorado Animal Care and Use Committee.

In all experiments the training context was created by placing a conditioning chamber into one of two identical ice chests $(54 \mathrm{~L} \times 30 \mathrm{~W} \times 27 \mathrm{H}, \mathrm{cm})$ with white interiors. This chamber was made of transparent Plexiglas and rectangular in shape (30 $\mathrm{L} \times 20 \mathrm{~W} \times 10 \mathrm{H}, \mathrm{cm})$. The top was made of wired mesh. The floor of the conditioning chamber was a stainless steel shock grid with rods $(1.5 \mathrm{~mm}$ diameter) spaced $1.2 \mathrm{~cm}$ center to center (Model E10-30SR, Coulburn Instruments). Each grid was wired to a shock source and scrambler (Model H13-16, Coulbourn Instruments). A clear Plexiglas panel connected the diagonal corners of the chamber. This made the interior of the chest triangular in shape. The ice chest was illuminated by a 7 -watt, $120 \mathrm{~V}$ DC light dimmed so that the rat was just visible to an observer. The test room itself was not illuminated during either training or testing with this context. This "training context" will hereafter be termed Context A.

On the conditioning day rats were placed into the training context, A, and 2 min later they received a 2 -sec, $1.0-\mathrm{mA}$ footshock. A second shock was delivered 2 min later. Rats were tested for contextual fear 1, 7, or 15 d later in either the original training context (Context A) or the generalization context (Context B). Freezing, the rat's dominant defensive fear response, was the dependent variable. Freezing was defined as the absence of all movement, except respiration. In Experiment 1 the test session was $6 \mathrm{~min}$ and in Experiment 2 it was $3 \mathrm{~min}$.

The test for generalized contextual fear occurred in a different context, Context B. The interior of the test chamber of Context B was rectangular $(29 \mathrm{~L} \times 21 \mathrm{~W} \times 17 \mathrm{H}, \mathrm{cm})$ and the interior of the Igloo was illuminated with a $24 \mathrm{~V}$ DC clear light bulb. The floor of the generalization context was also the stainless steel shock grid. A background fan producing white noise was turned on when rats were tested in Context B. In Experiment 2, rats were also exposed to another context, Context $\mathrm{X}$, a mouse cage that shared no features with either Context A or Context B. The mouse cage was placed in a well-illuminated large room.

\section{Acknowledgments}

This research was supported by National Institutes of Health grant NIH RO1 MH61316 to J.W.R. The publication costs of this article were defrayed in part by payment of page charges. This article must therefore be hereby marked as "advertisement" in accordance with 18 USC section 1734 solely to indicate this fact.

\section{References}

Anagnostaras, S.G., Maren, S., and Fanselow, M.S. 1999. Temporally graded retrograde amnesia of contextual fear after hippocampal damage in rats: Within-subjects examination. J. Neurosci. 19: 1106-1114.

\section{Learning \& Memory}


Antoniadis, E.A. and McDonald, R.J. 2000. Amygdala, hippocampus, and discrimination fear conditioning to context. Behav. Brain Res. 108: $1-19$.

Bontempi, B., Laurent-Demir, C., Destrade, C., and Jaffard, R. 1999. Time-dependent reorganization of brain circuitry underlying long-term memory storage. Nature 400: 671-674.

Botvinick, M.M., Braver, T.S., Barch, D.M., Carter, C.S., and Cohen, J.D 2001. Conflict monitoring and cognitive control. Psychol. Rev. 108: 624-652.

Eysenck, H.J. 1968. A theory of the incubation of anxiety/fear responses. Behav. Res. Ther. 6: 309-321.

Fanselow, M.S. and Rudy, J.W. 1998. Convergence of experimental and developmental approaches to animal learning and memory processes. In Mechanistic relationships between development and learning: Beyond metaphor (eds. T. Carew et al.), pp. 15-29. Dahlem Workshop Report. John Wiley \& Sons Ltd., Chichester.

Fletcher, P.C. and Henson, R.N.A. 2001. Frontal lobes and human memory. Insights from functional neuroimaging. Brain 124: 849-881.

Frankland, P.W. and Bontempi, B. 2005. The organization of recent and remote memories. Nat. Rev. Neurosci. 6: 119-130.

Frankland, P.W., Cestari, V., Filipkowski, R.K., McDonald, R.J., and Silva, A.J. 1998. The dorsal hippocampus is essential for context discrimination but not for contextual conditioning. Behav. Neurosci. 112: $863-874$

Frankland, P.W., Bontempi, B., Talton, L.E., Kaczmarek, L., and Silva, A.J. 2004. The involvement of the anterior cingulate cortex in remote contextual fear memory. Science 304: 881-883.

Kim, J.J. and Fanselow, M. 1992. Modality-specific retrograde amnesia of fear. Science 256: 675-676.

Lehman, H., Lacanilaa, S., and Sutherland, R.S. Complete or partial hippocampal damage produces equivalent retrograde amnesia for recent and remote contextual fear memories. Eur. J. Neurosci. (in press).

Maren, S. 2001. Neurobiology of Pavlovian fear conditioning. Annu. Rev. Neurosci. 24: 897-931.

Maviel, T., Durkin, T.P., Menzaghi, F., and Bontempi, B. 2004. Sites of neocortical reorganization critical for remote memory. Science 305: 96-99.

McClelland, J.L., McNaughton, B.L., and O'Reilly, R.C. 1995. Why are there complementary learning systems in hippocampus and neocortex: Insights from the successes and failures of connectionist models of learning and memory. Psychol. Rev. 102: 419-457.

Miyashita, Y. 2004. Cognitive memory: Cellular and network machinaries and their top-down control. Science 306: 435-440.
O'Reilly, R.C., Braver, T.S., and Cohen, J.D. 1999. A biologically based computational model of working memory. In Models of working memory: Mechanisms of active maintenance and executive control (eds. A. Miyake and P. Shah), pp. 375-411. Cambridge University Press, New York.

Ranganath, C., Cohen, M.X., Dam, C., and Desposito, M. 2004. Inferior temporal, prefrontal, and hippocampal contributions to visual working memory maintenance and associative memory retrieval. $J$. Neurosci. 24: 3917-3943.

Riccio, D.C. and Ebner, D.L. 1981. Post-acquisition modifications of memory. In Information processing in animals: Memory mechanisms (eds. N.E. Spear and R.R. Miller), pp. 291-317. Lawrence Erlbaum Associates, Hillsdale, NJ.

Riccio, D.C., Ackil, J.K., and Burch-Vernon, A. 1992. Forgetting of stimulus attributes: Methodological implications for assessing associative phenomena. Psychol. Bull. 112: 433-445.

Rosas, J.M. and Bouton, M.E. 1997. Additivity of the effects of retention interval and context change on latent inhibition: Toward resolution of the context forgetting paradox. J. Exp. Psychol. Anim. Behav. Process. 23: 283-294.

Rudy, J.W., Huff, N.C., and Matus-Amat, P. 2004. Understanding contextual fear conditioning: Insights from a two-process model. Neurosci. Biobehav. Rev. 28: 675-685.

Rudy, J.W., Biedenkapp, J.C., and O'Reilly, R.C. 2005. Prefrontal cortex and the organization of recent and remote memories: An alternative view. Learn. Mem. 12: 445-446.

Squire, L.R. and Alvarez, P. 1995. Retrograde amnesia and memory consolidation: A neurobiological perspective. Curr. Opin. Neurobiol. 5: $169-177$.

Squire, L.R., Cohen, N.J., and Nadel, L. 1984. The medial temporal region and memory consolidation: A new hypothesis. In Memory consolidation (ed. E.P.G. Weingartner), pp. 185-210. Erlbaum, Hillsdale, NJ.

Teyler, T.J. and DiScenna, P. 1986. The hippocampal memory indexing theory. Behav. Neurosci. 100: 147-154.

Wiltgen, B.J., Sanders, M.J., Anagnostaras, S.G., Sage, J.R., and Fanselow, M.S. 2006. Context fear learning in the absence of the hippocampus. J. Neurosci. 26: 5484-5491.

Zhou, Y.L. and Riccio, D.C. 1994. Pretest cuing can alleviate the forgetting of contextual stimulus attributes. Learn. Motiv. 12: $233-244$.

Received December 8, 2006; accepted in revised form January 17, 2007. 


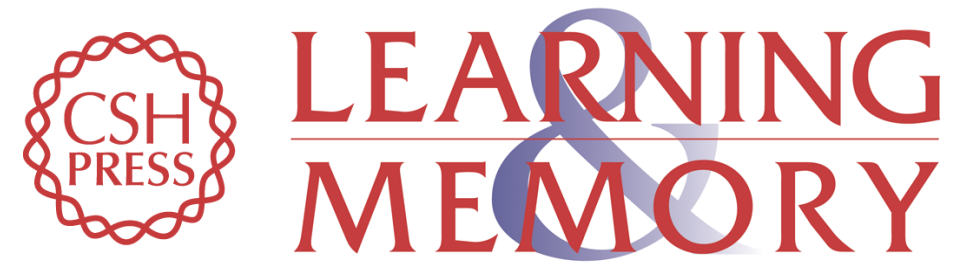

\section{Context preexposure prevents forgetting of a contextual fear memory: Implication for regional changes in brain activation patterns associated with recent and remote memory tests}

Joseph C. Biedenkapp and Jerry W. Rudy

Learn. Mem. 2007, 14:

Access the most recent version at doi:10.1101//m.499407

References This article cites 23 articles, 8 of which can be accessed free at: http://learnmem.cshlp.org/content/14/3/200.full.html\#ref-list-1

License

Email Alerting

Receive free email alerts when new articles cite this article - sign up in the box at the Service top right corner of the article or click here. 